\title{
Improved demonstration of immunohistochemical prognostic markers for survival in follicular lymphoma cells
}

Francisca I Camacho ${ }^{1}$, Carmen Bellas ${ }^{2}$, Cesáreo Corbacho ${ }^{2}$, Alexia Caleo ${ }^{3}$, Reyes Arranz-Sáez ${ }^{4}$, Jimena Cannata ${ }^{4}$, Javier Menárguez ${ }^{5}$, Lydia Sánchez-Verde ${ }^{6}$, Leocricia González-Camacho ${ }^{7}, \mathrm{M}^{\mathrm{a}}$ Elena Pérez-Martín ${ }^{8}$, Miguel A Martínez-González ${ }^{8}$, Tomás Álvaro ${ }^{9}$, Manuela Mollejo ${ }^{10}$, Carmen Ruíz-Marcellán ${ }^{11}$, Carlos Montalbán ${ }^{7}$ and Miguel A Piris ${ }^{6}$

${ }^{1}$ Department of Pathology, Hospital Universitario de Getafe, Madrid, Spain; ${ }^{2}$ Department of Pathology, Hospital Puerta de Hierro-Majadahonda, Madrid, Spain; ${ }^{3}$ Biomorphological and Functional Sciences Department, 'Federico II' University, Naples, Italy; ${ }^{4}$ Department of Haematology, Hospital de La Princesa, Madrid, Spain; ${ }^{5}$ Department of Pathology, Hospital General Universitario Gregorio Marañón, Madrid, Spain; ${ }^{6}$ Lymphoma Group, Molecular Pathology Program, Centro Nacional de Investigaciones Oncológicas (CNIO), Madrid, Spain; ${ }^{7}$ Department of Pathology, Hospital Ramón y Cajal, Madrid, Spain; ${ }^{8}$ Department of Pathology, Hospital Universitario 12 de Octubre, Madrid, Spain; ${ }^{9}$ Department of Pathology, Hospital Verge de la Cinta, Tortosa, Tarragona, Spain; ${ }^{10}$ Department of Pathology, Hospital Virgen de la Salud, Toledo, Spain and

${ }^{11}$ Department of Pathology, Hospital Universitario Vall d'Hebron, Barcelona, Spain

Follicular lymphoma (FL) is one of the most common forms of the low-grade non-Hodgkin's lymphoma in adults, with a characteristic translocation, $t(14 ; 18)(q 32 ; q 21)$ that deregulates the expression of the $B C L 2$ gene. The clinical course of FL patients is variable, whereby a subset of patients survive for long periods even without relapses, whereas the majority have frequent relapses with shorter survival. We have analyzed a series of 186 FLs, studying the correlation between clinical outcome and the tumor cell expression of a set of immunohistochemical markers, using an automated procedure for tissue microarrays to reduce the subjectivity of scoring. The results identified several markers associated with differences in overall survival (OS) in univariate analyses, such as Cyclin E, Mdm2, CD10, p21, IgD, Bcl-xL, CD30, and E2F6. Cases with a higher level of expression of Cyclin E, Mdm2, p21, IgD, Bcl-xL, CD30, and E2F6 were associated with a significantly shorter OS. On the other hand, strong CD10 expression was linked to a significantly better outcome. A Cox model was then constructed, integrating the Follicular Lymphoma International Prognostic Index (FLIPI) score and a restricted selection of three immunohistochemical markers: Cyclin E, Mdm2, and CD10 expression. A potentially useful finding is that the integrated FLIPI plus immunohistochemical model can be used to identify a subset of 26 patients (almost $20 \%$ of the total series), with a survival probability of $100 \%$ at 5 years. This not only confirms that a group of FL cases may have a very good clinical course, but also indicates that this group can be identified using this integrated clinical and immunohistochemical approach.

Modern Pathology (2011) 24, 698-707; doi:10.1038/modpathol.2010.237; published online 14 January 2011

Keywords: follicular lymphoma; immunohistochemistry; prognostic; tissue microarray

Correspondence: Dr FI Camacho, MD, PhD, Department of Pathology, Hospital Universitario de Getafe, Carretera de Toledo, km 12.5, E-28905: Getafe, Madrid, Spain.

E-mail: ficamacho.hugf@salud.madrid.org

Received 27 May 2010; revised 9 September 2010; accepted 23 November 2010; published online 14 January 2011
Follicular lymphoma (FL) is one of the most common forms of the low-grade non-Hodgkin's lymphoma in adults. It is characterized by the presence of the translocation $t(14 ; 18)(q 32 ; q 21)$, which deregulates the expression of the $B C L 2$ gene. Clinical course in FL patients is variable, whereby 
some patients survive for long periods even without relapses, although the majority has frequent relapses with shorter survival.

Currently, the prognosis of FL patients is essentially based on the clinical parameters of the International Prognostic Index (IPI) or the Follicular Lymphoma International Prognostic Index (FLIPI), ${ }^{1}$ the latter having being designed specifically for this type of B-cell lymphoma. Many immunohistochemical and molecular markers have been considered to be potential prognostic markers in FL, carrying information from the tumoral cells or the microenvironment, but none of them are used in routine clinical diagnosis. Significant and potentially useful data have recently emerged regarding the role of the microenvironment in the prognosis of $\mathrm{FL}^{2-7}$ Less attention has been paid to the role of tumoral cell markers in the prognosis of FL patients, partially because of the difficulty of obtaining reproducible measurements of protein expression from paraffinembedded samples.

Previous studies have demonstrated that predictors based on a number of biomarkers that can be obtained at diagnosis in tissue microarrays (TMAs) have the potential to improve the prediction of overall survival (OS) in FL. ${ }^{8}$ Here, we have analyzed a large series of FL samples to identify potentially useful markers that could be merged in a biological prognostic model. The series has an exceptionally long follow-up, with a mean of 173 months, thus making it possible to use OS as the end point. Markers were selected on the basis of previous studies of the pathogenesis or prognosis of FL. To increase the reliability of the data generated, the immunohistochemical markers were analyzed with a quantitative image analysis system that improves the reproducibility of the results. ${ }^{9}$

\section{Materials and methods}

This series included 186 cases of nodal FL collected from seven institutions, diagnosed between 1980 and 2002, with clinical follow-up and available clinical information. All cases were reviewed by two pathologists (FIC and CB) and reclassified according to the WHO classification. ${ }^{10}$ The grade of every case was evaluated simultaneously by both the pathologists, and disagreements were resolved through a joint review using a multiheaded microscope. The only criteria used for patient selection were the diagnosis confirmation, the availability of a paraffin block for TMA analysis, and of the appropriate clinical information. The study was conducted under the supervision of the ethical committee of the Hospital La Paz, Madrid, Spain. This same series has also been used to analyze the impact of the reactive microenvironment in the prognosis of FL. ${ }^{5,6}$

We used a tissue arrayer device (Beecher Instruments, Silver Springs, MD, USA) to construct eight TMA blocks, according to conventional protocols. ${ }^{9}$
All cases were subjected to routine $\mathrm{H} \& \mathrm{E}$ staining and were histologically reviewed according to the criteria of the WHO classifications. ${ }^{10}$ Two representative $1.5-\mathrm{mm}$ diameter cylinders, from two preselected areas in the paraffin block, were included in each case, along with 16 separate controls to ensure the quality, reproducibility, and homogenous staining of the slides. Selected controls included reactive lymph nodes and tonsils, and paraffinembedded FL cell lines.

Immunohistochemistry was performed using standard procedures ${ }^{9}$ to demonstrate the expression of 28 selected proteins (Table 1) related to apoptosis control (extrinsic and intrinsic pathways), cell cycle, B-cell differentiation, and signaling. The association of these molecules with survival probability was evaluated.

Fluorescence in situ hybridization was performed on TMA slides according to standard procedures. ${ }^{11}$ LSI IGH/BCL2 dual color, dual fusion translocation probe (Vysis, Downers Grove, IL, USA) was used to detect the BCL2 translocation. Samples were considered positive when a distinctive pattern of one red, one green, and two red/green (yellow) fusion signals was present in more than $10 \%$ of nuclei.

All hybridized TMAs were scanned using the Bacus Laboratories Incorporated Slide Scanner system (Bacus Laboratories, Lombard, IL, USA), which uses three CCD RGB sensor optically coupled to a microscope. TMAs were quantitatively scored

Table 1 Range and mean \pm s.d. of protein expression of 28 markers analyzed on TMA

\begin{tabular}{lcccrr}
\hline Marker & $\mathrm{N}$ & $\begin{array}{c}\text { Minimum } \\
(\%)\end{array}$ & $\begin{array}{c}\text { Maximum } \\
(\%)\end{array}$ & $\begin{array}{r}\text { Mean } \\
(\%)\end{array}$ & $\begin{array}{r}\text { s.d. } \\
(\%)\end{array}$ \\
\hline p53 & 176 & 0.01 & 79.98 & 5.36 & 11.883 \\
Bax & 170 & 0.00 & 93.83 & 7.56 & 21.324 \\
BCL2 & 170 & 0.01 & 89.93 & 42.29 & 24.687 \\
MIB1 & 181 & 3.18 & 50.39 & 20.82 & 8.515 \\
pRB & 176 & 0.00 & 46.10 & 3.24 & 6.088 \\
Survivin & 163 & 0.01 & 91.74 & 30.87 & 27.032 \\
BCL6 & 179 & 0.01 & 65.24 & 13.66 & 16.211 \\
Cyclin A & 169 & 0.02 & 89.41 & 7.20 & 11.037 \\
Cyclin B1 & 170 & 0.00 & 77.02 & 1.36 & 7.229 \\
MUM1 & 178 & 0.07 & 58.60 & 6.01 & 9.475 \\
CD38 & 177 & 0.02 & 79.06 & 16.35 & 16.441 \\
Cyclin D3 & 159 & 0.00 & 26.23 & 0.56 & 2.251 \\
CDK6 & 169 & 0.01 & 65.94 & 6.46 & 11.144 \\
CD27 & 171 & 0.00 & 75.48 & 16.58 & 18.766 \\
PU 1 & 179 & 0.00 & 72.91 & 9.50 & 14.164 \\
Cyclin E & 179 & 0.00 & 4.09 & 0.07 & 0.348 \\
E2F6 & 182 & 0.11 & 48.65 & 8.90 & 8.525 \\
CDK2 & 177 & 0.00 & 76.68 & 3.28 & 8.526 \\
Caspase 3a & 181 & 0.01 & 16.29 & 0.96 & 1.883 \\
p27 & 182 & 0.08 & 96.27 & 55.65 & 24.723 \\
Mdm2 & 178 & 0.00 & 12.44 & 0.80 & 1.744 \\
CD30 & 179 & 0.00 & 24.88 & 0.47 & 2.313 \\
Bcl-xL & 182 & 0.72 & 63.80 & 15.95 & 10.984 \\
CD10 & 182 & 0.05 & 90.56 & 49.78 & 27.368 \\
Skp2 & 174 & 0.00 & 50.27 & 2.40 & 5.426 \\
p21 & 185 & 0.00 & 49.24 & 3.05 & 5.998 \\
IgD & 170 & 0.08 & 87.20 & 17.95 & 19.310 \\
p18 & 165 & 0.05 & 45.34 & 10.38 & 8.435 \\
\hline & & & & & \\
\hline
\end{tabular}


with TMAscore v.1.0 image analysis software, which uses WebSlide v.1.0 virtual slides (all Bacus Laboratories). The system is designed to quantify immunohistochemical staining using images obtained by scanning, and specific parameters fixed for each marker. Protein expression was measured as the percentage of the total core areas that was positively stained. The scanning system measured the total area occupied by cells, avoiding fibrosis or other negligible areas. The data were then exported to spreadsheets or database management systems for further analysis (Figure 1). The higher of the two values of positive expression was selected from each case.

To relate the expression of the different markers to the clinical data, the series was divided into quintiles with respect to the expression of each protein (Tables 1 and 2).

Overall survival was the end point of all statistical analyses. Only deaths attributable to the disease or to toxicity of treatment were considered. Survival curves were performed using the Kaplan-Meier estimator and compared using the log-rank test. Cox univariate analysis was also performed independently for each variable to estimate relative risk (Exp (B)). Values of $P<0.05$ were considered significant. Survival probability was assessed by a Cox multivariate analysis that included all the variables with a value of $P<0.1$. An individual risk for each patient was assigned using the value of $X-\beta$, which was obtained from the integrated markers (protein-based survival predictor) in the Cox multivariate analysis. All patients were ranked with respect to $X-\beta$ value and divided into equal groups before applying the Kaplan-Meier estimator and log-rank test. All statistical analyses were carried out using SPSS v.15.0 (Chicago, IL, USA).

\section{Results}

\section{Patients}

The series was comprised of 186 cases of nodal FL collected from seven institutions, diagnosed between 1980 and 2002, with clinical follow-up and available clinical information (Table 1). The median age of patients was 56 years, with a range from 17 to 85 years, of whom 82 were men and 104 were women. The FLIPI score was obtained for 150 cases and the distribution with respect to this index was as follows: low-risk, 46 cases; intermediate-risk, 59 cases; and high-risk, 45 cases. The IPI score was calculated for 170 cases with the following distribution: 80 low-risk cases, 82 intermediate-risk cases, and 8 high-risk cases. The series included 35 patients with low clinical Ann-Arbor stages (I-II) and 147 patients with high clinical stages (III-IV).

With respect to histological grade, 80 cases were considered as grade 1, 57 cases as grade 2, 36 cases as grade $3 \mathrm{a}$, and 13 cases as grade $3 \mathrm{~b}$. No significant differences in OS were found between these four groups.

OS ranged from 1 to 250 months and the mean follow-up was 173 months. A total of 46 patients died because of the disease. Transformation to diffuse large B-cell lymphoma (DLBCL) was observed in six cases, with four of them dying of the disease.

Approximately, $36 \%$ of informative cases (41 out of 114) were negative for the $(14 ; 18)(q 32 ; q 21)$ translocation. A trend towards better prognosis was observed in negative $\mathrm{t}(14 ; 18)(\mathrm{q} 32 ; \mathrm{q} 21)$ cases, with six out of 41 patients dying of the disease, in contrast with $21 / 73$ cases positive for $t(14 ; 18)$ (q32;q21). However, these differences were not statistically significant. These data have been previously published. ${ }^{12}$

Treatment protocols varied considerably within the series, over time, and among institutions. The protocols included 'watch and wait' approach, different regimens of chemotherapy, radiotherapy, and bone marrow transplantation as first-line therapy. A total of 74 patients were treated with cyclophosphamide, vincristine, and prednisone (CVP), including 61 patients treated with CVP plus immunomodulator (58 with $\alpha$-interferon and three with anti-CD20 monoclonal antibody). Other nonaggressive combinations without alkylating agent were used as first-line therapy in five cases. Aggressive combination chemotherapy was the therapy option in 86 cases, out of whom 65 received cyclophosphamide, doxorubicin, vincristine, and prednisone (CHOP), including six CHOP plus $\alpha$ interferon and five CHOP plus anti-CD20 monoclonal antibody. The other 21 patients were treated by chemotherapy with alkylating agents (C-MOOP, etc). Monotherapy with cyclophosphamide or clorambucil plus prednisone or leukeran plus prednisone was given to eight patients, and localized radiation therapy was used to treat four patients. A 'watch and wait' approach was taken in nine patients. In this series, eight patients were initially treated with anti-CD20 monoclonal antibody, whereas eight patients were treated with autologous stem cell transplantation or non-myeloablative allogeneic stem cell transplantation. The different therapeutic approaches made it essential to collect samples from different clinical institutions and over a relatively long period.

\section{Survival Analysis}

Univariate analysis identified several clinical parameters with the capacity to predict OS (Table 3). The group of patients aged 60 years or more, at high AnnArbor stage, bone marrow involvement, and high performance status had significantly shorter OS, measured by the clinical FLIPI and IPI scores.

However, several markers were associated with differences in OS in univariate analyses, such as 

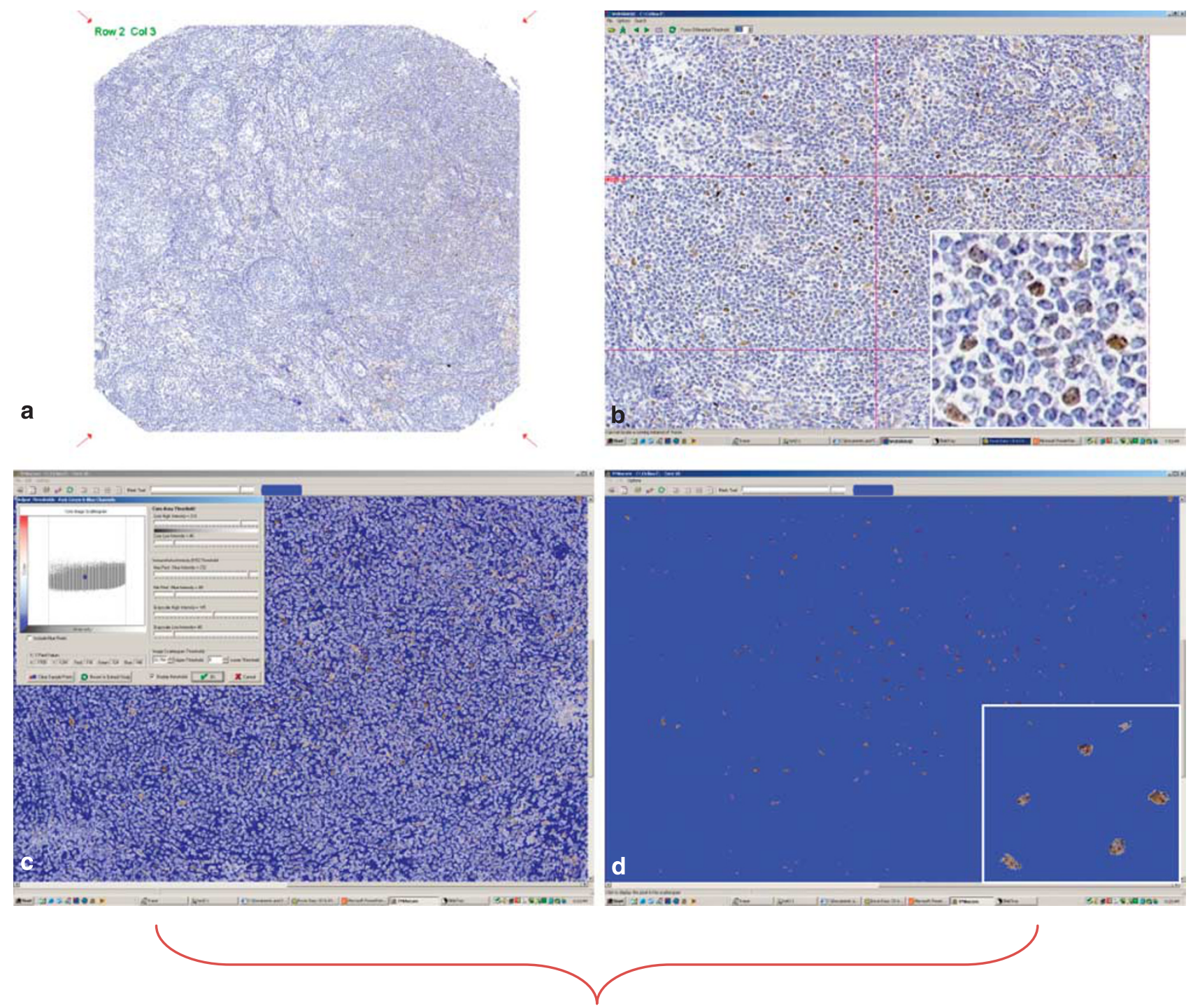

\begin{tabular}{|cccccc|}
\hline Row & Column & Core & Area $\left(\mu^{2}\right)$ & $\mathrm{IHC}$ Area $\left(\mu^{2}\right)$ & $\%$ IHC Area \\
1 & 1 & 1 & 214376.38 & 119.78 & 0.06 \\
1 & 2 & 2 & 419355.80 & 0.69 & 0.00 \\
1 & 3 & 3 & 521691.44 & 973.38 & 0.19 \\
1 & 4 & 4 & 748635.76 & 439.03 & 0.06 \\
1 & 5 & 5 & 272804.76 & 64884.04 & 23.78 \\
1 & 6 & 6 & 281193.11 & 31.77 & 0.01 \\
1 & 7 & 7 & 661330.59 & 52.60 & 0.01 \\
1 & 8 & 8 & 866127.03 & 2009.59 & 0.23 \\
1 & 9 & 9 & 398874.99 & 769.74 & 0.19 \\
1 & 10 & 10 & 3573.90 & 0 & 0 \\
2 & 1 & 20 & 810029.93 & 79.86 & 0.01 \\
2 & 2 & 19 & 696669.13 & 62.32 & 0.01 \\
2 & 3 & 18 & 616259.34 & 370.64 & 0.06 \\
2 & 4 & 17 & 396946.82 & 8783.12 & 2.21 \\
\hline
\end{tabular}

Figure 1 Scheme of quantification system using TMAscore v.1.0 image analysis software, which uses WebSlide v.1.0 virtual slides (all from Bacus Laboratories). The image shows the quantitative expression of a nuclear marker (Mdm2) in a selected core. (a) Low-power view of the immunostaining. (b) High-power view, with an insert showing a selected field. (c) Measuring the area occupied by cells. (d) Positive immunostaining in the selected field. (e) Example of quantitative results.

Cyclin E, Mdm2, CD10, p21, IgD, Bcl-xL, CD30, and E2F6. Those cases with a higher level of expression of cyclin E, Mdm2, p21, IgD, Bcl-xL, CD30, and E2F6 were associated with a significantly shorter OS. On the other hand, strong CD10 expression was linked to a significantly better outcome. We did not find any differences between the five groups defined by Ki67 expression (Table 4). 
Table 2 Mean \pm s.d. of each quintile of the expression of the eight markers with differences in OS probability

\begin{tabular}{|c|c|c|c|c|c|c|c|}
\hline Marker & Groups & 1 & 2 & 3 & 4 & 5 & Total \\
\hline Cyclin E & $\underset{N}{\operatorname{Mean} \pm \mathrm{s} . \mathrm{d}}$ & - & $\begin{array}{c}0.00 \pm 0.000 \\
70\end{array}$ & $\begin{array}{c}0.01 \pm 0.00 \\
41\end{array}$ & $\begin{array}{c}0.03 \pm 0.009 \\
30\end{array}$ & $\begin{array}{c}0.3 \pm 0.7 \\
38\end{array}$ & $\begin{array}{c}0.08 \pm 0.3 \\
179\end{array}$ \\
\hline Mdm2 & $\underset{N}{\operatorname{Mean} \pm \mathrm{s} . \mathrm{d}}$ & $\begin{array}{c}0.01 \pm 0.01 \\
32\end{array}$ & $\begin{array}{c}0.06 \pm 0.02 \\
38\end{array}$ & $\begin{array}{c}0.2 \pm 0.09 \\
37\end{array}$ & $\begin{array}{c}0.6 \pm 0.1 \\
36\end{array}$ & $\begin{array}{c}3.1 \pm 2.9 \\
35\end{array}$ & $\begin{array}{c}0.8 \pm 1.7 \\
178\end{array}$ \\
\hline CD10 & $\underset{N}{\operatorname{Mean} \pm \mathrm{s} . \mathrm{d}}$ & $\begin{array}{c}5.5 \pm 6.1 \\
36\end{array}$ & $\begin{array}{c}35.5 \pm 8.7 \\
37\end{array}$ & $\begin{array}{c}57.1 \pm 5.7 \\
36\end{array}$ & $\begin{array}{c}69.9 \pm 2.9 \\
37\end{array}$ & $\begin{array}{c}80.8 \pm 3.5 \\
36\end{array}$ & $\begin{array}{c}49.8 \pm 27.4 \\
182\end{array}$ \\
\hline p21 & $\underset{N}{\operatorname{Mean} \pm \text { s.d. }}$ & $\begin{array}{c}0.07 \pm 0.05 \\
39\end{array}$ & $\begin{array}{c}0.4 \pm 0.1 \\
35\end{array}$ & $\begin{array}{c}1 \pm 0.3 \\
37\end{array}$ & $\begin{array}{c}2.4 \pm 0.7 \\
37\end{array}$ & $\begin{array}{c}11.4 \pm 9.5 \\
37\end{array}$ & $\begin{array}{c}3 \pm 6 \\
185\end{array}$ \\
\hline $\operatorname{IgD}$ & $\underset{N}{\operatorname{Mean} \pm \mathrm{s} . \mathrm{d}}$ & $\begin{array}{c}1.2 \pm 0.7 \\
34\end{array}$ & $\begin{array}{c}4.3 \pm 1.1 \\
34\end{array}$ & $\begin{array}{c}10.2 \pm 2.1 \\
34\end{array}$ & $\begin{array}{r}23.5 \pm 5 \\
34\end{array}$ & $\begin{array}{c}50.5 \pm 14.6 \\
34\end{array}$ & $\begin{array}{c}17.9 \pm 19.3 \\
170\end{array}$ \\
\hline Bcl-xL & $\underset{N}{\operatorname{Mean} \pm \mathrm{s} . \mathrm{d}}$ & $\begin{array}{c}4.7 \pm 1.7 \\
36\end{array}$ & $\begin{array}{c}9.4 \pm 1.2 \\
37\end{array}$ & $\begin{array}{c}13.5 \pm 1.4 \\
36\end{array}$ & $\begin{array}{c}19.1 \pm 1.8 \\
37\end{array}$ & $\begin{array}{c}33.2 \pm 10.7 \\
36\end{array}$ & $\begin{array}{c}15.9 \pm 11 \\
182\end{array}$ \\
\hline CD30 & $\underset{N}{\operatorname{Mean} \pm \text { s.d. }}$ & $\begin{array}{c}0 \pm 0 \\
30\end{array}$ & $\begin{array}{c}0.01 \pm 0.005 \\
44\end{array}$ & $\begin{array}{c}0.04 \pm 0.01 \\
35\end{array}$ & $\begin{array}{c}0.1 \pm 0.03 \\
35\end{array}$ & $\begin{array}{c}2.2 \pm 4.9 \\
35\end{array}$ & $\begin{array}{c}0.5 \pm 2.3 \\
179\end{array}$ \\
\hline E2F6 & $\underset{N}{\operatorname{Mean} \pm \mathrm{s} . \mathrm{d}}$ & $\begin{array}{c}1.6 \pm 0.7 \\
37\end{array}$ & $\begin{array}{c}3.6 \pm 0.5 \\
36\end{array}$ & $\begin{array}{c}6.2 \pm 0.9 \\
36\end{array}$ & $\begin{array}{c}10 \pm 1.6 \\
37\end{array}$ & $\begin{array}{c}23.2 \pm 8.2 \\
36\end{array}$ & $\begin{array}{c}8.9 \pm 8.5 \\
182\end{array}$ \\
\hline
\end{tabular}

Table 3 Outcome and survival analysis according to group as defined by different clinical parameters and histological grading

\begin{tabular}{|c|c|c|c|c|c|}
\hline Parameter & $\mathrm{N}$ total & $\mathrm{N}$ & 5 -year OS (\%) & 10-year OS (\%) & Log-rank test, $\mathrm{P}$ \\
\hline Gender & 186 & & & & NS \\
\hline Female & & 104 & 88 & 71 & \\
\hline Male & & 82 & 82 & 57 & \\
\hline Age & 185 & & & & 0.005 \\
\hline$<60$ years & & 109 & 91 & 74 & \\
\hline$\geq 60$ years & & 76 & 78 & 48 & \\
\hline Cell type & 186 & & & & NS \\
\hline Grade 1 & & 80 & 86 & 60 & \\
\hline Grade 2 & & 57 & 83 & 68 & \\
\hline Grade 3a & & 36 & 84 & 69 & \\
\hline Grade $3 b$ & & 13 & 100 & 67 & \\
\hline Ann-Arbor stages & 182 & & & & 0.001 \\
\hline I-II & & 35 & 97 & 97 & \\
\hline III-IV & & 147 & 83 & 56 & \\
\hline Number of nodal sites & 186 & & & & NS \\
\hline $0-4$ & & 119 & 88 & 72 & \\
\hline$>4$ & & 67 & 82 & 53 & \\
\hline Bone marrow involvement & 172 & & & & 0.01 \\
\hline Absence & & 80 & 88 & 77 & \\
\hline Presence & & 92 & 82 & 51 & \\
\hline Serum $L D H$ & 171 & & & & NS \\
\hline$\leq \mathrm{ULN}$ & & 144 & 85 & 66 & \\
\hline$>\mathrm{ULN}$ & & 27 & 88 & 58 & \\
\hline Hemoglobin level & 157 & & & & NS \\
\hline$\geq 120 \mathrm{~g} / \mathrm{l}$ & & 126 & 89 & 64 & \\
\hline$<120 \mathrm{~g} / \mathrm{l}$ & & 31 & 89 & 75 & \\
\hline FLIPI & 150 & & & & 0.002 \\
\hline $0-1$ & & 46 & 97 & 89 & \\
\hline$\geq 2$ & & 104 & 86 & 55 & \\
\hline IPI & 170 & & & & 0.0001 \\
\hline $0-1$ & & 80 & 93 & 80 & \\
\hline$\geq 2$ & & 90 & 77 & 47 & \\
\hline Follow-up (months) & 186 & & & & \\
\hline Mean $^{\mathrm{a}}$ & & 173 & & & \\
\hline Total range & & $1-251$ & 86 & 65 & \\
\hline DOD & & 46 & & & \\
\hline
\end{tabular}

Abbreviations: DOD, death of disease; FLIPI, Follicular Lymphoma International Prognostic Index; IPI, International Prognostic Index; LDH, lactate dehydrogenase; NS, not statistically significant; OS, overall survival; ULN, upper limit normal.

${ }^{\mathrm{a}}$ Median not reached.

Significant $P$-values are in bold. 
Cyclin E showed a range of protein expression (\%) from 0.00 to 4.09, with a mean of 0.07 ; Mdm2 expression ranged from 0.00 to 12.44 , with a mean of 0.80; CD10 expression ranged from 0.05 to 90.56, with a mean of 49.80; p21 showed a range of expression from 0.00 to 49.24 , with a mean of 3.05 ; the expression of $\operatorname{IgD}$ protein ranged from 0.08 to 87.20 , with a mean of 17.95; Bcl-xL expression ranged from 0.72 to 63.80 with a mean of 15.90 ; CD30 protein expression ranged from 0.00 to 24.90 with a mean expression of 0.50; and E2F6 protein expression ranged from 0.11 to 48.65 , with a mean of 8.90 (Table 2).

The Cox multivariate analysis showed that Cyclin E, Mdm2, and CD10 expression (Figure 2) were markers with a predictive capacity that was independent of FLIPI (Table 4).
To represent the survival curves defined in this way, the individual risk for each patient was assigned using the value of $\mathrm{X}-\beta$, which was obtained from the integrated markers (IHC survival predictor or protein model) identified in the Cox multivariate analysis. All patients were ranked with respect to $\mathrm{X}-\beta$ value, and divided into equal groups before applying the Kaplan-Meier estimator and carrying out a log-rank test (Figure 3). A 5-year survival for patients in the low-risk group was $95 \%$ and for highrisk group was $77 \%$, the difference being statistically significant (log-rank test; $P<0.0001$ ).

This protein expression model was then used for both the low and intermediate-high FLIPI groups using the Kaplan-Meier method, and proved to be a significant prognostic marker for both. The expression of the three markers divided the low-risk FLIPI

Table 4 Cox univariate and multivariate analyses

\begin{tabular}{|c|c|c|c|c|c|c|c|c|c|}
\hline \multirow[t]{3}{*}{ Marker } & \multicolumn{5}{|c|}{ Univariate analysis } & \multicolumn{4}{|c|}{ Multivariate analysis } \\
\hline & \multirow[b]{2}{*}{$\mathrm{N}$} & \multicolumn{3}{|c|}{$95 \%$ CI for $\operatorname{Exp}(B)$} & \multirow[b]{2}{*}{$\mathrm{P}$} & \multicolumn{3}{|c|}{$95 \%$ CI for $\operatorname{Exp}(B)$} & \multirow[b]{2}{*}{$\mathrm{P}$} \\
\hline & & $\operatorname{Exp}(B)$ & Lower & Upper & & $\operatorname{Exp}(B)$ & Lower & Upper & \\
\hline Cyclin E & 68 vs 111 & 2.8322 & 1.5342 & 5.2284 & 0.0009 & 2.0302 & 1.079 & 3.8198 & 0.0281 \\
\hline M̆dm2 & 146 vs 32 & 12.1814 & 1.6744 & 88.6216 & 0.0135 & 10.3266 & 1.4109 & 75.5812 & 0.0215 \\
\hline CD10 & 73 vs 109 & 1.964 & 1.0776 & 3.5793 & 0.0275 & 2.0678 & 1.1038 & 3.8737 & 0.0233 \\
\hline p21 & 74 vs 111 & 3.0518 & 1.6645 & 5.5953 & 0.0003 & - & - & - & - \\
\hline $\mathrm{IgD}$ & 34 vs 136 & 2.6001 & 1.3959 & 4.843 & 0.0026 & - & - & - & - \\
\hline Bcl-xL & 36 vs 146 & 1.9517 & 1.0343 & 3.6826 & 0.0390 & - & - & - & - \\
\hline CD30 & 70 vs 109 & 2.3942 & 1.3125 & 4.3672 & 0.0044 & - & - & - & - \\
\hline E2F6 & 36 vs 146 & 2.3176 & 1.2414 & 4.3269 & 0.0083 & - & - & - & - \\
\hline
\end{tabular}

Significant $P$-values are in bold.

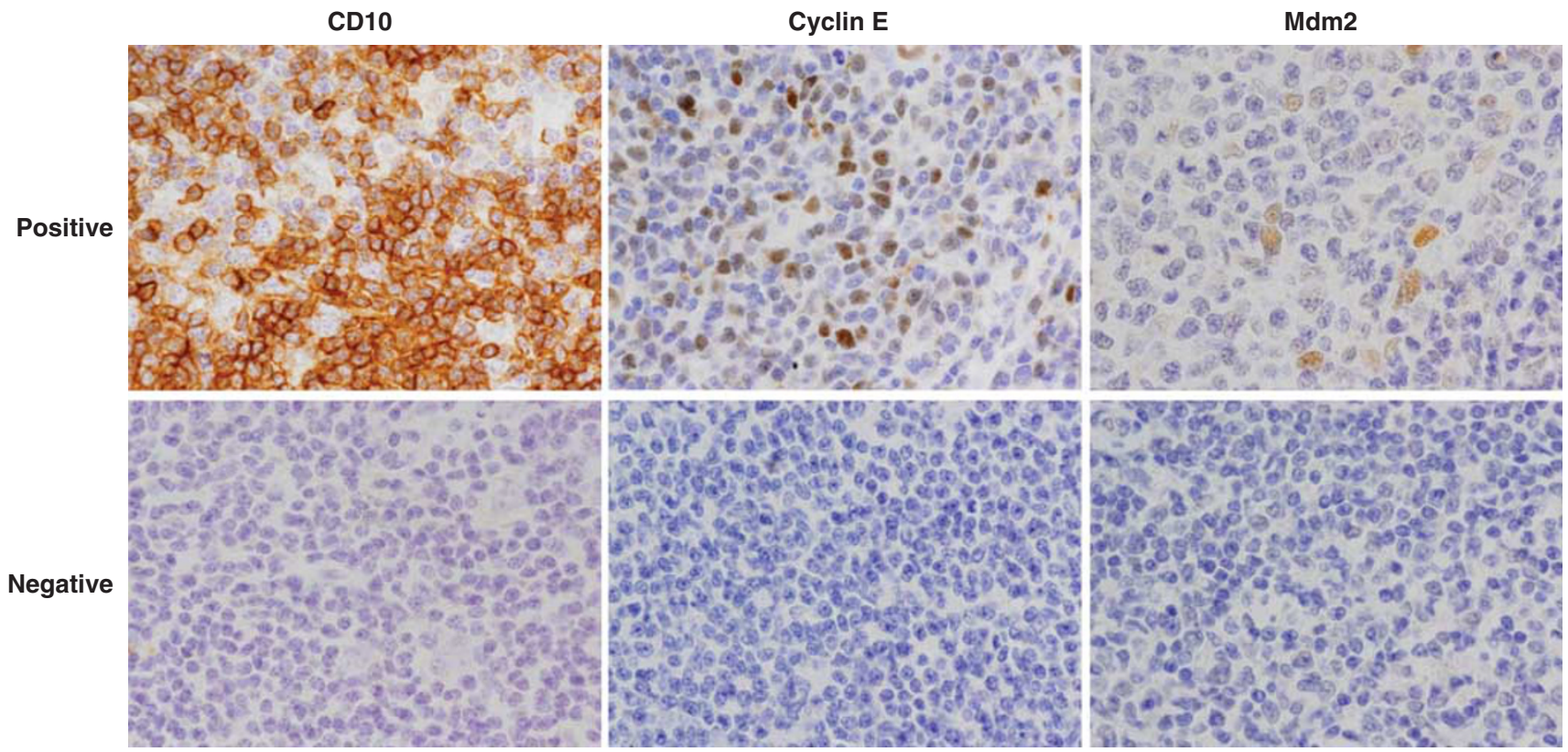

Figure 2 Immunohistochemical expression of markers with differences in overall survival and included in the Cox multivariate analysis $(\times 40)$. 
group into two groups with 100 vs 93\% survival probabilities at 5 years and 100 vs $68 \%$ at 10 years (log-rank test; $P<0.05)$. This was also the case for the intermediate-high risk FLIPI groups in which two groups were identified with 97 vs $81 \%$ and 76 VS $39 \%$ survival probabilities at 5 and 10 years, respectively, were found (log-rank test; $P<0.01$ ) (Figure 4).

No significant differences in OS were observed between FL histological grades 1-3 (Table 3). However, the protein expression model was able to discriminate different survival probabilities in low-grade (grades 1 and 2) and high-grade (grade 3) histological groups. Cases with low-grade histology and a low protein score had 95 and $82 \%$ OS probabilites at 5 and 10 years, respectively, in

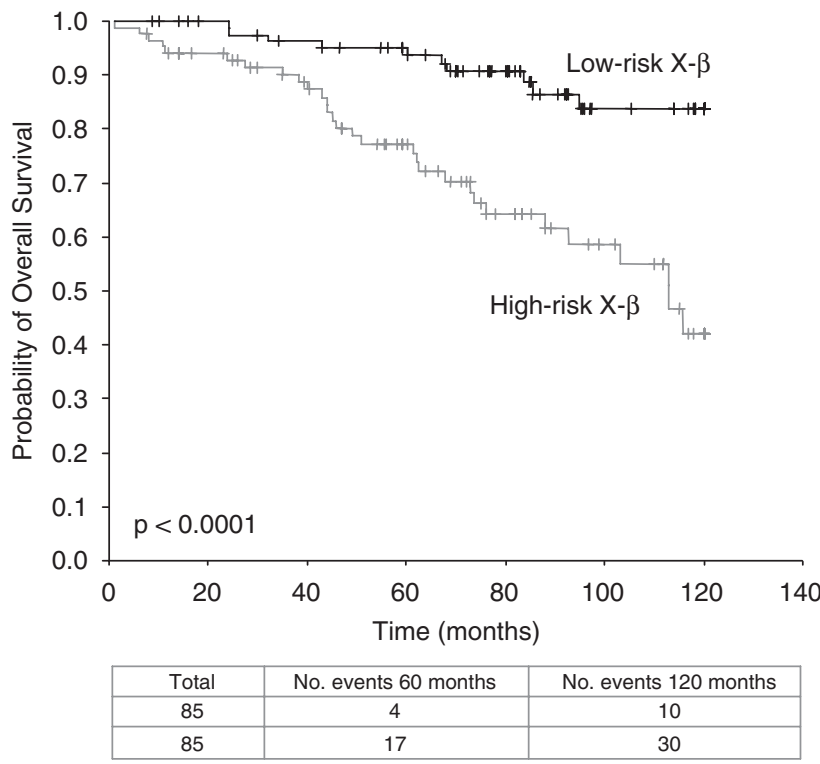

Figure 3 Survival curves of FL patients divided into two equal groups by integration of the three markers identified in the multivariate analysis ( $\mathrm{X}-\beta$ assigned on the basis of the outcome of the Cox analysis). contrast with 72 and $40 \%$ in cases with low-grade histology and high protein score. High histological grade cases and low protein score showed 95 and $89 \%$ survival probability at 5 and 10 years, respectively, whereas cases with the same histological grade but high protein score had 81 and $48 \%$ survival probability at 5 and 10 years, respectively (log-rank; $P<0.0001$ ) (Figure 5).

Protein score was also independent of the therapy received. To analyze the interaction between the protein score and the therapy, the series were divided into non-aggressive and aggressive therapy groups. The distribution of protein expression scores revealed two non-aggressive therapy subgroups with 94 vs $71 \%$ survival probabilities at 5 years and 94 vs $49 \%$ at 10 years. Cases treated with aggressive therapy were divided using the protein score into a group with $95 \mathrm{vs} 87 \%$ at 5 years and 77 vs $29 \%$ at 10 years (log-rank test; $P<0.0001$ ) (Figure 6).

\section{Discussion}

In this study, we identified a set of markers expressed by neoplastic cells that were associated with low OS in FL patients. These included Cyclin E, Mdm2, CD10, p21, IgD, Bcl-xL, CD30, and E2F6. Their expression was estimated using an automated procedure that reduces the subjectivity of scoring. Some of these markers, or others related with them, have already been shown to be prognostic markers in FL patients. ${ }^{13,14}$ A Cox model was then built integrating FLIPI and a restricted selection of three markers: Cyclin E, Mdm2, and CD10 expression.

A potentially useful finding is that the integrated FLIPI plus IHC model was able to identify a subset of $26 \mathrm{FL}$ patients (almost 20\% of the entire series) with a survival probability of $100 \%$ at 5 years. This not only confirms that a group of FL cases may have a very good clinical course, but also indicates that
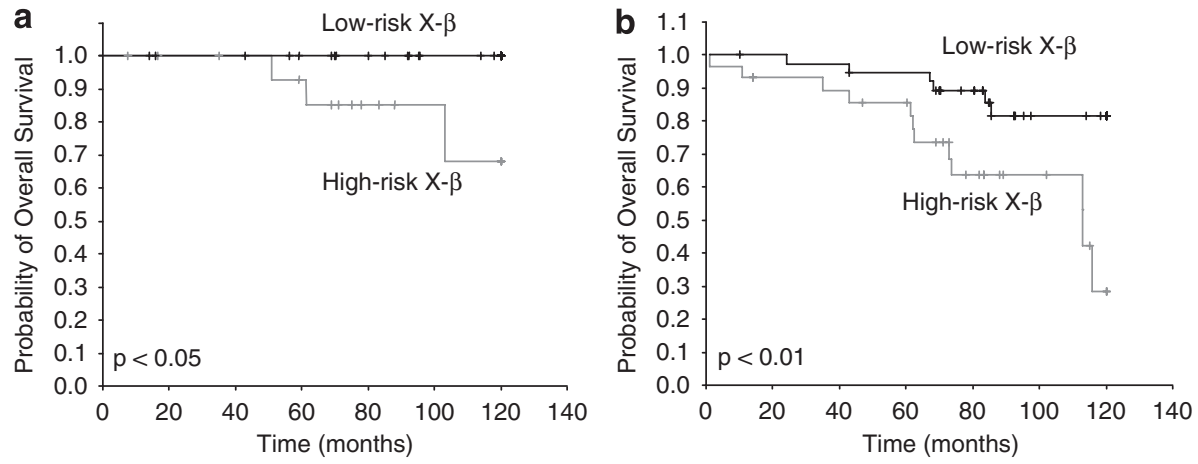

\begin{tabular}{|c|c|c|}
\hline & Total & No. events \\
\hline Low-risk FLIPI & 43 & 3 \\
\hline Low-risk X- $\beta$ & 26 & 0 \\
\hline High-risk X- $\beta$ & 17 & 3 \\
\hline
\end{tabular}

\begin{tabular}{|r|c|c|}
\hline & Total & No. events \\
\hline Intermediate-high-risk FLIPI & 93 & 24 \\
\hline Low-risk X- $\beta$ & 39 & 5 \\
\hline High-risk X- $\beta$ & 54 & 19 \\
\hline
\end{tabular}

Figure 4 Survival curves of two groups defined by X- $\beta$ (protein model) in low-risk FLIPI (a) and intermediate-high-risk FLIPI series of patients (b). Log-rank test; $P=0.001$. 

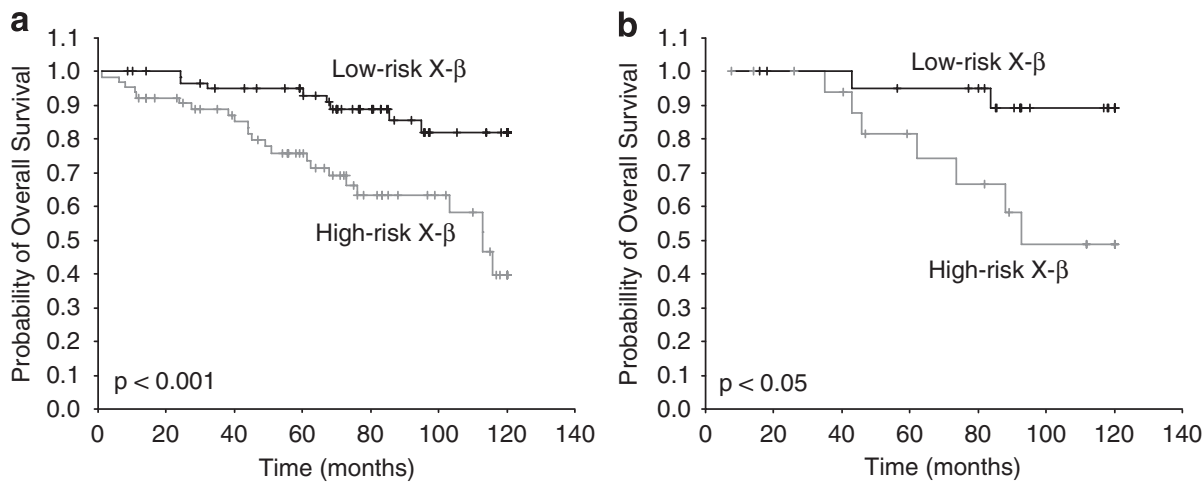

\begin{tabular}{|c|c|c|}
\hline & Total & No. events \\
\hline Low Grade & 127 & 31 \\
\hline Low-risk X- $\beta$ & 62 & 8 \\
\hline High-risk X- $\beta$ & 65 & 23 \\
\hline
\end{tabular}

\begin{tabular}{|l|c|c|}
\hline & Total & No. events \\
\hline High Grade & 43 & 9 \\
\hline Low-risk X- $\beta$ & 23 & 2 \\
\hline High-risk X- $\beta$ & 20 & 7 \\
\hline
\end{tabular}

Figure 5 Survival curves of two groups defined by X- $\beta$ (protein model) in low-histological grade (a) and high-grade series of FL cases (b). Log-rank test; $P<0.0001$.
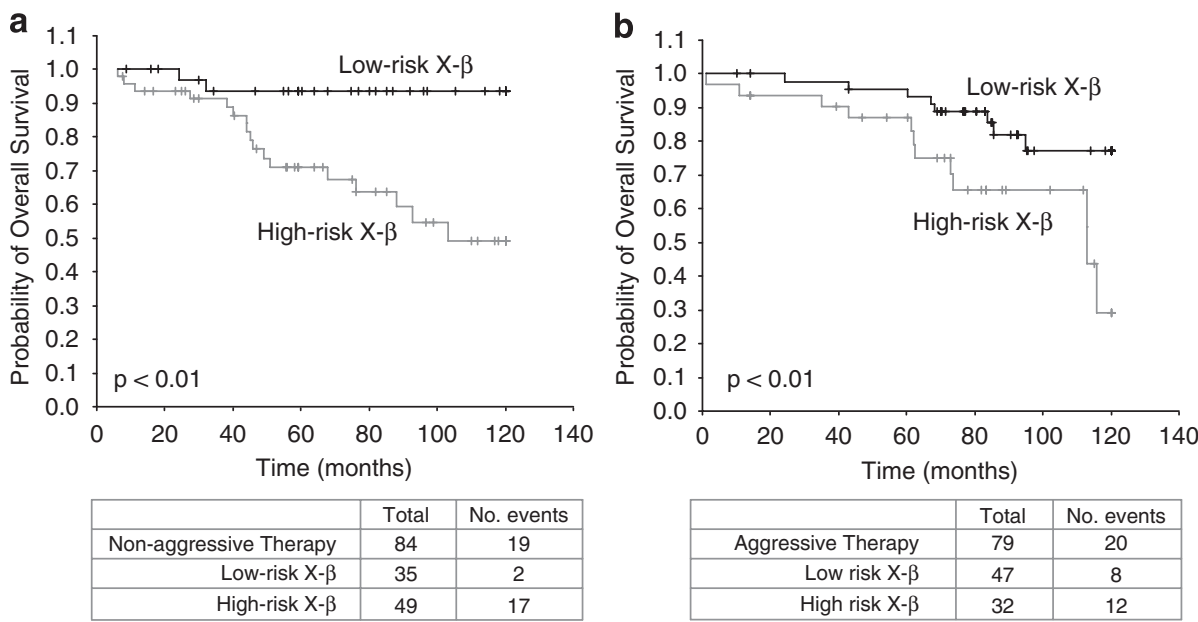

Figure 6 Survival curves of two groups defined by X- $\beta$ (protein model) in non-aggressive chemotherapy (a) and aggressive chemotherapy (b) groups of patients. Log-rank test; $P=0.001$.

this group can be identified using this integrated clinical and IHC approach.

Cyclin $\mathrm{E}$ is a cyclin involved in the initiation of DNA replication, genomic stability, and the centrosome cycle. The cyclin E/CDK2 complex is able to activate the cascade of molecules needed in S-phase and that are critical in G1-to-S transitions during the cell cycle. In fact, on the basis of results of studies of human cancers such as breast cancer, leukemia, and lymphoma, it is considered to be an oncoprotein. ${ }^{15}$ Our findings reveal higher expression of cyclin E and its kinase partner, CDK2, in a group of patients with poorer outcome, with cyclin $\mathrm{E}$ being an independent factor identified in the multivariate analysis.

CD10 is considered to be a marker of follicular center B-cell differentiation. This prognostic marker has previously been described. ${ }^{13} \mathrm{PU}-1$ is a transcription factor that is also involved in B-cell differentiation, and a significant positive association has been observed between a high level of GC antigen expression (PU-1, CD27, CD75, CD20, Bcl-6, CD10, etc.) and longer OS. ${ }^{16}$ In our study, a significantly better clinical outcome was related with strong expression of CD10, independent of FLIPI, suggesting that the strong GC differentiation signature in FL could also be defined a group of lymphomas with better prognosis, similarly to the situation described in DLBCL. ${ }^{17}$

The Mdm2 oncoprotein is a cellular inhibitor of p53 that is frequently deregulated in human cancer. Univariate analysis of our series revealed a highly statistically significant shorter OS for higher levels of $\mathrm{Mdm} 2$ protein expression, the better prognostic group having almost negative $\mathrm{Mdm} 2$ expression $(P=0.01)$. Moreover, this protein was included in the final multivariate model. Mdm2 protein overexpression has previously been reported in B-cell lymphoma and in transformed FL cases, independent of TP53 status. ${ }^{18-24}$ 
A potentially related finding is the strongly raised level of p53 expression observed in 5\% of the series, which is associated with shorter OS, and confirms the results about the prognostic value of the p53 mutation. ${ }^{25}$ We have not included p53 in the final model, as the proportion of positive cases was substantially below the required threshold $(20 \%$ cases).

This study has focused on the features of the tumoral cells, rather than other cell components of the FL microenvironment that have been already demonstrated to have a prognostic significance. FL genesis, progression, and treatment escape represent a scenario of complex relationship between molecules and signal from tumoral and non-tumoral cells. ${ }^{2}$ Further analyses integrating both sources of data are still to be performed, but seem likely to enrich the results shown here.

Histological grading in the diagnosis of FL is still routinely included in the pathological report, following the WHO recommendations. ${ }^{10}$ In this series, no significant difference in OS was found according to the histological grading. Moreover, the integration of the protein model and grade demonstrated that the model was independent of the histological grading, with strong OS predictive capacity for both low and high-grade groups.

This series is not based on a standardized treatment, and includes patients treated with different protocols, reflecting the absence of any standard approach to the treatment of FL. However, the model here identified was independent of the treatment, when patients were divided into two groups considering the different therapeutic options. Prospective prognostic studies in FL are hampered by the relatively indolent clinical course that requires long follow-up. In contrast, this retrospective study offers the opportunity of including patients with a long follow-up, thereby increasing the capacity of the study to identify prognostic markers. In this study we have followed the model of patient selection previously used in the development of the FLIPI. ${ }^{1}$

The study does not consider whether changes in treatment protocols can modify these prognostic markers, as has been shown for some microenvironment markers. ${ }^{26}$ The specific effect of Rituximab should be analyzed after a longer follow-up than that currently available.

\section{Acknowledgements}

This study is supported by grants from the Ministerio de Ciencia e Innovación (RETICS, FIS PI052742, PI052800, INT07/028, SAF2008-03871) and the Servicio de Salud de Castilla la Mancha (SESCAM 06047-00, FISCAM GCS-2006-C/06), Spain. The Spanish Tumour Bank Network did an excellent job collecting all the samples, a complicated task that was superbly managed by Laura Cereceda.

\section{Disclosure/conflict of interest}

Authors declare no conflict of interest.

\section{References}

1 Solal-Celigny P, Roy P, Colombat P, et al. Follicular lymphoma international prognostic index. Blood 2004;104:1258-1265.

2 Dave SS, Wright G, Tan B, et al. Prediction of survival in follicular lymphoma based on molecular features of tumor-infiltrating immune cells. N Engl J Med 2004; 351:2159-2169.

3 Farinha P, Masoudi H, Skinnider BF, et al. Analysis of multiple biomarkers shows that lymphoma-associated macrophage (LAM) content is an independent predictor of survival in follicular lymphoma (FL). Blood 2005;106:2169-2174.

4 Carreras J, Lopez-Guillermo A, Fox BC, et al. High numbers of tumor-infiltrating FOXP3-positive regulatory $\mathrm{T}$ cells are associated with improved overall survival in follicular lymphoma. Blood 2006;108: 2957-2964.

5 Alvaro T, Lejeune M, Camacho FI, et al. The presence of STAT1-positive tumor-associated macrophages and their relation to outcome in patients with follicular lymphoma. Haematologica 2006;91:1605-1612.

6 Alvaro T, Lejeune M, Salvado MT, et al. Immunohistochemical patterns of reactive microenvironment are associated with clinicobiologic behavior in follicular lymphoma patients. J Clin Oncol 2006;24:5350-5357.

7 Farinha P, Al-Tourah A, Gill K, et al. The architectural pattern of FOXP3-positive $\mathrm{T}$ cells in follicular lymphoma is an independent predictor of survival and histologic transformation. Blood 2009;115:289-295.

8 Korenberg MJ, Farinha P, Gascoyne RD. Predicting survival in follicular lymphoma using tissue microarrays. Methods Mol Biol 2007;377:255-268.

9 Garcia JF, Camacho FI, Morente M, et al. Hodgkin and Reed-Sternberg cells harbor alterations in the major tumor suppressor pathways and cell-cycle checkpoints: analyses using tissue microarrays. Blood 2003; 101:681-689.

10 Swerdlow S, Campo E, Harris N, et al. WHO Classification of Tumours of Haematopoietic and Lymphoid Tissues. IARC Press: Lyon, 2008.

11 Buno I, Nava P, Alvarez-Doval A, et al. Lymphoma associated chromosomal abnormalities can easily be detected by FISH on tissue imprints. An underused diagnostic alternative. J Clin Pathol 2005;58:629-633.

12 Diaz-Alderete A, Doval A, Camacho F, et al. Frequency of BCL2 and BCL6 translocations in follicular lymphoma: relation with histological and clinical features. Leuk Lymphoma 2008;49:95-101.

13 Bilalovic N, Blystad AK, Golouh R, et al. Expression of bcl-6 and CD10 protein is associated with longer overall survival and time to treatment failure in follicular lymphoma. Am J Clin Pathol 2004;121: 34-42.

14 Zhao WL, Daneshpouy ME, Mounier N, et al. Prognostic significance of bcl-xL gene expression and apoptotic cell counts in follicular lymphoma. Blood 2004;103:695-697.

15 Keyomarsi K, Tucker SL, Buchholz TA, et al. Cyclin E and survival in patients with breast cancer. $\mathrm{N}$ Engl J Med 2002;347:1566-1575. 
16 Torlakovic EE, Bilalovic N, Golouh R, et al. Prognostic significance of PU.1 in follicular lymphoma. J Pathol 2006;209:352-359.

17 Alizadeh AA, Eisen MB, Davis RE, et al. Distinct types of diffuse large B-cell lymphoma identified by gene expression profiling. Nature 2000;403:503-511.

18 Wang P, Lushnikova T, Odvody J, et al. Elevated Mdm2 expression induces chromosomal instability and confers a survival and growth advantage to B cells. Oncogene 2008;27:1590-1598.

19 Moller MB, Nielsen O, Pedersen NT. Oncoprotein MDM2 overexpression is associated with poor prognosis in distinct non-Hodgkin's lymphoma entities. Mod Pathol 1999;12:1010-1016.

20 Moller MB, Nielsen O, Pedersen NT. Frequent alteration of MDM2 and p53 in the molecular progression of recurring non-Hodgkin's lymphoma. Histopathology 2002;41:322-330.

21 Sanchez E, Chacon I, Plaza MM, et al. Clinical outcome in diffuse large B-cell lymphoma is dependent on the relationship between different cell-cycle regulator proteins. J Clin Oncol 1998;16:1931-1939.
22 Pagnano KB, Vassallo J, Lorand-Metze I, et al. p53, Mdm2, and c-Myc overexpression is associated with a poor prognosis in aggressive nonHodgkin's lymphomas. Am J Hematol 2001;67: 84-92.

23 Davies AJ, Lee AM, Taylor C, et al. A limited role for TP53 mutation in the transformation of follicular lymphoma to diffuse large B-cell lymphoma. Leukemia 2005;19:1459-1465.

24 Eischen CM, Weber JD, Roussel MF, et al. Disruption of the ARF-Mdm2-p53 tumor suppressor pathway in Myc-induced lymphomagenesis. Genes Dev 1999;13: 2658-2669.

25 O'Shea D, O'Riain C, Taylor C, et al. The presence of TP53 mutation at diagnosis of follicular lymphoma identifies a high-risk group of patients with shortened time to disease progression and poorer overall survival. Blood 2008;112:3126-3129.

26 de Jong D, Koster A, Hagenbeek A, et al. Impact of the tumor microenvironment on prognosis in follicular lymphoma is dependent on specific treatment protocols. Haematologica 2009;94:70-77. 